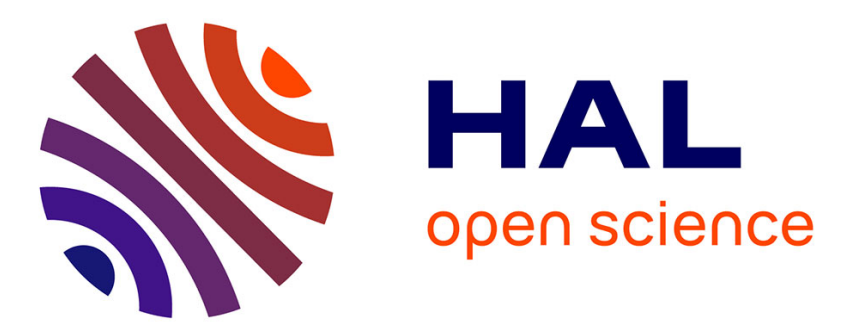

\title{
Des récits entre droit au séjour et droit à l'incohérence : l'accompagnement juridique dans les associations de défense des droits des étrangers
}

\author{
Laura Odasso
}

\section{- To cite this version: \\ Laura Odasso. Des récits entre droit au séjour et droit à l'incohérence : l'accompagnement juridique dans les associations de défense des droits des étrangers. CORPS : Revue Interdisciplinaire, 2020, N¹8 (1), pp.67-78. 10.3917/corp1.018.0067 . hal-03119269}

\author{
HAL Id: hal-03119269 \\ https://hal.science/hal-03119269
}

Submitted on 23 Jan 2021

HAL is a multi-disciplinary open access archive for the deposit and dissemination of scientific research documents, whether they are published or not. The documents may come from teaching and research institutions in France or abroad, or from public or private research centers.
L'archive ouverte pluridisciplinaire HAL, est destinée au dépôt et à la diffusion de documents scientifiques de niveau recherche, publiés ou non, émanant des établissements d'enseignement et de recherche français ou étrangers, des laboratoires publics ou privés. 


\title{
DES RECITS ENTRE DROIT AU SÉJOUR ET DROIT A L'INCOHÉRENCE : L'ACCOMPAGNEMENT JURIDIQUE DANS LES ASSOCIATIONS DE DEFENSE DES DROITS DES ETRANGERS
}

\author{
Laura Odasso
}

«La nature la plus profonde des individus est à fleur de peau:

la peau des autres »

(Goffman, 1973: 338)

Depuis 2015, la vision de l'immigration comme une crise sans précédent et l'attention démesurée portée aux «nouvelles » conflictualités qu'elle met en exergue risquent de faire oublier la réalité ordinaire de la migration et son historicité. Or, les nouveaux venus s'ajoutent - avec des dynamiques propres à la conjoncture historique et socio-politique - aux nombreux migrants en situation de précarité et administrativement absents qui habitent nos villes depuis longtemps. Produits des politiques migratoires qui, dès les années 90 au moins, tendent à les «illégaliser» (Dauvergne, 2008), ces individus interagissent de temps en temps avec le dispositif d'immigration afin de reprendre en main leurs «carrières de papier ${ }^{1} »($ Spire, 2005 : 34-35). Leurs changements de situation, les dispositions de la loi d'immigration en mutation continue et le modus operandi de leur mise en œuvre selon les territoires et les époques peuvent, en effet, leur offrir des opportunités pour sortir de l'impasse juridico-administrative. Pour ces individus, l'accès à une voie de régularisation implique la construction d'un récit destiné à prouver qu'ils remplissent les conditions pour demander le «droit à être là » et à émerger en tant que « personnes » (Dal Lago, 1999). Ce « récit de présence »-comme je le qualifie dans cette contribution pour souligner sa portée de reconnaissance d'une existence peu visible, voire invisible, mais parfois bruyante (Laacher, 2009), et d'ouverture du droit au séjour - doit assumer la forme d'une narration de soi non ambiguë et objectivable. Le demandeur élabore une version de son histoire migratoire et y associe des preuves (par exemple, documents privés, certificats administratifs) pouvant matérialiser sa présence passée sur le territoire et justifier son droit d'y rester. Ces documents sont soumis à l'évaluation de l'administration de l'immigration - en France, le service des étrangers de la préfecture - qui statue sur le séjour. Elle vérifie la véracité de la situation qui motive la demande sur la base des informations fournies par le demandeur et de celles présentes dans les bases des données administratives et policières qui le concernent. Ce procédé est le produit d'une interaction bureaucratique définie, une injonction socio-institutionnelle à (s')élaborer un récit de soi (Butler, 2007) pour exister administrativement.

En amont de la rencontre avec l'administration, des travailleurs sociaux, des juristes, des bénévoles d'associations de défense des droits des étrangers, des avocats et des informateurs informels, parfois des migrants, participent de ses dynamiques. Ces acteurs - que nous appellerons « intermédiaires du droit », pour signaler leurs compétences juridiques et savoirfaire pratiques de traduction en vue de l'accès aux droits - contribuent à la construction des récits «adaptés » et au rassemblement des preuves adéquates pour monter les dossiers de régularisation. Ce faisant, ils encadrent la mise en récit des personnes migrantes à la demande institutionnelle (Fassin et al., 2013). De fait, ils donnent leur avis sur la version de l'histoire migratoire de ces demandeurs et sur les chances que leur dossier soit accepté et, donc, mérite d'être suivi de près et étoffé pour maximiser son succès lors de l'évaluation administrative. Dans cette contribution, nous allons nous pencher sur la rencontre avec ces intermédiaires. Je me propose en effet d'éclairer, dans les situations d'accompagnement juridique associatif, les 
circonstances de la mise en récit des carrières de migrants qui ont peu de chances d'être régularisés, selon le droit en place. Je vais donc parler, non pas de tous ceux qui s'appuient sur l'accompagnement juridique dans les associations de défense des droits des étrangers, mais de ces usagers particuliers plongés dans l'insécurité administrative et figures de l'exclusion radicale (Fassin et Morice, 2001). Dans un contexte de suspicion accrue de détournement des procédures administratives à des fins migratoires, leurs dossiers ont de véritables difficultés à franchir le seuil de l'administration et nécessitent, donc, une action juridique pointue et spécifique. Or, d'une part, le malaise social et parfois physique et psychique de ces personnes migrantes, en situation administrative irrégulière, défie le travail des intermédiaires du droit et, s'autre part, en retour, ces personnes migrantes sont mises au défi par les intermédiaires au travers d'un travail relationnel normatif propre aux procédures en matière d'immigration. Ces interactions avec des personnes migrantes en souffrance se situent à la frontière entre obtention du droit au séjour et reconnaissance d'un " droit à l'incohérence » (Butler, 2007 ; Fœssel, 2009). Les effets que les marques de la loi inscrites sur les corps et dans la psyché de ces personnes «non-régularisables » produisent dans les situations d'intermédiation juridique invitent à repenser autrement ce travail, ainsi que le positionnement du chercheur dans ce terrain sensible.

L'article s'appuie sur une partie du matériau ethnographique issu d'une enquête qui visait à étudier les expériences bureaucratiques et juridiques de migrants, ainsi que les rôles des intermédiaires du droit. Dans ce but, engagée comme bénévole, j'ai effectué une période d'immersion d'un an - entre 2016 et 2017 - dans une association de défense des droits des étrangers dans une grande ville française ${ }^{2}$. De cette position, il ne s'agissait pas uniquement d'observer les interactions entre les membres de l'association et les personnes migrantes en vue de la résolution de leur situation administrative, mais de « faire de la participation mon instrument de connaissance » (Faavret-Saada, 2009: 153). Une démarche d'auto-observation et de réflexion sur ma pratique de terrain a accompagné cette implication. Je l'introduirai dans l'analyse afin d'éclairer en quoi ma propre subjectivité et ma corporéité, en tant que femme étrangère, européenne et blanche, privilégiée du fait d'être immigrée par choix, influent sur la construction dialogique du récit des personnes rencontrées.

\section{LA PERMANENCE JURIDIQUE ASSOCIATIVE : LES LIMITES D'UN ESPACE « PROTEGE »}

Un nombre croissant d'acteurs du tiers secteur assure la prise en charge juridico-sociale des personnes migrantes pour faciliter leurs rencontres avec les guichets préfectoraux, mais se trouve aussi à contenir leur malaise psychosocial. Ces acteurs, qui effectuent un travail de gestion des corps migrants qui s'apparente de celui de l'État (Pette, 2014), tout en n'étant pas spécialistes des savoirs de la psyché, doivent faire face aussi à l' « intériorité » de leurs interlocuteurs troublés par les effets inattendus de la migration. L'accompagnement juridique bascule vers une forme d'assistance humanitaire qui vise à susciter chez les bénéficiaires la compréhension et l'acceptation là où les mesures légales ne peuvent plus rien faire. Malgré ça, les migrants qui s'y réfèrent nécessitent l'expertise de ces intermédiaires du droit et leur travail de négociation à la marge de l'État.

Des migrants s'adressent à l'association, que j'ai observée, pour se préparer au mieux aux rencontres administratives en vérifiant la faisabilité de leur démarche et l'adéquation des pièces à joindre à leurs dossiers administratifs. Certains s'y renseignent sur leurs droits avant d'entamer toute action, tandis que d'autres s'y rendent lorsque des blocages surviennent dans leurs procédures en cours. D'autres, dont la demande est en traitement, passent pour boire une boisson chaude et soulager l'attente en échangeant avec des bénévoles ou d'autres bénéficiaires. D'autres encore s'y présentent régulièrement, apparemment sans un objectif précis ou pour poser des questions aux bénévoles. L'hétérogénéité des trajectoires 
personnelles et des carrières migratoires (Martiniello et Rea, 2011) imprègne la salle d'attente où la plupart des personnes ont en commun l'espoir de trouver des réponses à leur situation, voire sa reconnaissance. Cette dernière se manifeste par une sortie de la subordination statutaire que leur impose la loi d'immigration grâce à l'acquisition d'un statut administratif et, plus largement, à l'écoute et la prise au sérieux de leur histoire. En réalité, par l'entrée de l'aide juridique, cet espace associatif offre, avant tout, un territoire protégé où des bénévoles opèrent aussi un travail de soin émotionnel de ces personnes en demande d'aide que les blessures juridiques exposent à d'autres blessures intimes et sociales souvent bien plus lourdes (Jamoulle, 2009).

Lors des permanences ouvertes au public, une partie de l'équipe de l'association, dans des espaces en retrait, se consacre à l'accompagnement juridique individuel et, une autre partie, passe son temps en première ligne, dans la salle d'attente. Ici, les bénévoles s'occupent d'échanger avec les visiteurs, de saisir leurs demandes et de les traiter si elles ne relèvent pas du juridique proprement dit. Ce premier tri permet de soulager les autres bénévoles. L'association a un seul salarié. La majorité de bénévoles sont des femmes françaises, blanches, retraitées qui ont une formation et ont travaillé dans le social, l'éducation nationale et le droit. On compte quelques juristes encore en activité et des jeunes, français et européens, en stage de droit ou de travail social, dont la plupart se définissent militants. Toutefois, pour des bénévoles - interrogés pendant le travail ethnographique - leur engagement pour la cause des étrangers ne suffit plus pour surmonter les écueils que leur imposent le régime d'immigration en place et les moyens mêmes de l'association. Ils doívent régulièrement remettre à jour leurs connaissances, car le droit des étrangers et sa pratique évoluent rapidement, mais cette formation ne fait que leur dévoiler les limites légales de leur possibilité d'agir. Les politiques et les administrations de l'immigration cherchent à dissuader les étrangers, les sélectionnent et les précarisent. Or, ces acteurs associatifs se retrouvent à faire le « sale boulot» (Lhuilier, 2005). Sans les moyens suffisants et contraintes par les lois en place, leurs tâches deviennent de plus en plus palliatives. Ils se retrouvent impuissants confrontés aux carrières migratoires et aux corps marginaux, « inutiles » à l'économie politique et morale (Fassin, 2001:7) et, rejetés par l'État qui refuse de les intégrer dans la nation. Le temps et les forces humaines qui manquent brident leur travail lorsque les personnes migrantes qui se trouvent dans les coulisses du système augmentent considérablement. Cela affecte également les interactions entre bénévoles et migrants et la perception que ces derniers ont à l'égard de l'aide associatif.

\section{LE BINOME D'ACCOMPAGNEMENT JURIDIQUE : LA MISE EN RECIT DU POINT DE VUE DES ACTEURS}

Les bénéyoles qui s'occupent de l'accompagnement juridique effectuent, d'abord, un entretien voué à la compréhension minutieuse des étapes personnelles, administratives, professionnelles et familiales des personnes en demande d'aide, ainsi qu'un diagnostic de la matérialité des leurs carrières migratoires. Cette interaction entraîne un processus intersubjectif et dialogique entre aidés et aidants : une phase de mise en ordre préalable des faits et de partage de la trame narrative essentielle pour une mise en récit efficace. À la différence des demandeurs d'asile et de certains conjoints des couples binationaux qui sont auditionnés à propos de leurs histoires, les autres demandeurs sont plutôt invités à produire de « bonnes » preuves et à échanger informellement avec les guichetiers qui réceptionnent leur dossier. C'est sur la base de ces matériaux, qui appartiennent au sujet, mais ne sont pas subjectifs (Ferraris, 2009), que l'administration statuera. La valeur de ces matériaux réside dans le travail de sens qui leur est associé, d'une part, par les personnes migrantes qui les inscrivent dans les étapes de leur récit de vie et, d'autre part, par tous les acteurs qui donnent un avis sur leur validité en matière d'ouverture des droits, y compris dans 
les associations. Tout détail pourra être pertinent pour entrevoir de pistes procédurales en vue de l'accession au séjour, « les dates, les démarches entamées, les réponses administratives reçues, les liens sur le territoire et la chronologie des événements doivent être précisés [...], on leur demande des certificats, des documents, des papiers pour comprendre et vérifier ce qu'ils nous racontent », dit Anne, assistante sociale à la retraite et bénévole depuis six ans. Elle m'explique les grandes lignes des démarches à suivre, le reste je l'apprendrai sur le tas dans un «binôme d'accompagnement». C'est ainsi que l'équipe appelle l'outil pratique d'apprentissage pour les nouveaux bénévoles qui, associés à des bénévoles plus anciens et plus experts, apprennent « comment se fait l'accompagnement » (Anne), et s'entraînent jusqu'à qu'ils se sentent assez autonomes pour recevoir eux-mêmes des personnes, voire pour former d'autres bénévoles.

Cette situation à trois individus - la personne migrante, le bénévole et moi, la bénévole(chercheure) - ayant des statuts divers et des pouvoirs asymétriques s'avère fournir une perspective d'observation pertinente pour comprendre les dynamiques d'une mise en récit dialogique et pour saisir comment ma présence, en tant que participante à la situation, affectait la dynamique et en était, à son tour, affectée. Je m'insérais, en effet, dans une situation où les ressources étaient interdépendantes et polarisées entre personnes migrantes en quête d'aide et bénévoles aidants, détenteurs de la connaissance. Dans leur interaction, de l'empathie trouve sa place notamment lorsque ces individus en co-présence acceptent leurs rôles respectifs et mettent en acte des stratégies pour se faire comprendre (Hollan et Throop, 2008).

À l'occasion d'un binôme d'accompagnement, Johan, l'un des seuls bénévoles de sexe masculin, juriste retraité et actif auprès de l'association depuis cinq ans, m'explique : «ici aussi finalement, on sélectionne un peu les gens, en fonction des chances de leur cas. » En utilisant « aussi », il se réfère à la préfecture, instance qui statue sur le droit au séjour en classifiant les demandeurs selon des catégories d'État. Les bénévoles associatifs ne peuvent que reprendre et réappliquer ces catégories, malgré leurs limites au vu des trajectoires complexes des migrants. À chaque catégorie correspondent des conditions et des preuves précises que ceux-ci doivent fournir. De fait, c'est moins ce qu'ils sont, que ce qu'ils racontent et prouvent, qui compte. «Nous avons de moins en moins de pistes à donner aux gens-là depuis longtemps, si leurs raisons et documents ne sont pas valables pour la préf' », affirme Johan qui a intériorisé le fonctionnement de cet accès au droit, par catégories, qui limite sont action. Cependant, il continue : « des personnes se représentent cycliquement. Ils sont chagrinés et malades dans l'âme, pas des malades qui peuvent se faire régulariser pour leur maladie, non, ils sont devenus fous ou déprimés du fait d'être ici dans ces conditions. » Avec ces mots, Johan signale les troubles psychiques des personnes migrantes qu'il lui arrive de rencontrer. Ses compétences juridiques ne suffisent pas toujours, et elles doivent céder le pas à une pratique de soin, « on les écoute, on les fait réfléchir, et on les envoie ailleurs chez des associations spécialisées » (Johan). Dans ce cas, en effet, face aux troubles, l'empathie, outil clé pour saisir les éléments les plus cachés des récits, montre ses limites, car ces personnes ont du mal à faire comprendre leurs besoins. Kirmayer (2008) soutient qu'une attitude empathique à l'égard des autres peut être maintenue même au moment où l'on ne comprend pas ou plus. Cependant, dans le cas qui nous occupe ici, cette empathie s'inscrit dans un contexte politique et social donné qui en biaise l'intelligibilité et qui façonne les possibilités de construire un récit de soi formellement acceptable. Les critères étatiques pour la concession du droit au séjour s'attachent davantage aux corps et à leur fonctionnalité, et imprègnent d'autres acteurs pris dans la gouvernance migratoire, y compris les bénévoles associatifs. Chez ceux avec qui j'ai travaillé, des représentations des carrières migratoires et ses effets physico-psychiques émergent et, associées aux contraintes pratiques, contribuent à développer des savoirs d'expérience en fonction des profils des bénéficiaires. 
Les personnes migrantes qui mettent à mal l'intersubjectivité de l'accompagnement juridique ont attiré mon attention pour deux raisons. Premièrement, la vulnérabilité et l'agitation qu'elles transmettaient et la difficulté à obtenir de leur part un récit intelligible ont instauré un non-dit - à investiguer - entre les bénévoles et moi à propos de la gestion des troubles existentiels et interactionnels dans l'accompagnement juridique. Deuxièmement, la rencontre avec ces personnes m'a demandé un effort spécifique, en tant que chercheure participante, entre projections et représentations de situations que j'observais et vivais.

Je développe ces deux points à l'aide des notes de terrain relatives au cas de Noah, prénom inventé d'une personne que j'ai suivi en binôme avec Johan. Noah, un homme de grande taille originaire des Comores, la quarantaine, aux cheveux bouclés et ébouriffés, est visiblement trop habillé pour la saison quand il arrive à la permanence. Dans la salle d'attente, il enlève et remet sa veste. Il est nerveux. Quand il essaie d'aborder des personnes en attente à l'accueil de la permanence, certaines le regardent avec méfiance, d'autres avec curiosité ou compassion au vu de leurs commentaires. Il a apporté un grand sac de sport usé, tout comme son pantalon, d'ailleurs. Il n'arrive pas à rester assis, il s'approche de la porte $\mathrm{d}$ accès aux espaces où les permanences individuelles se déroulent. Je ne suis pas capable de dire pourquoi il est là, aujourd'hui. Je faisais un exercice depuis que je conduisais mes observations en travaillant en alternance dans la salle d'attente pour accueillir les personnes, dans l'espace dédié à l'accompagnement juridique individuel avec un bénévole et en participant, ensuite, aux réunions collectives de débriefing entre bénévoles à la fin de l'après-midi de permanence. Je cherchais à deviner la raison de la venue de certains migrants et si cela correspondait au récit que j'imaginais être le leur. Puis, j'allais échanger avec certains d'entre eux et je cherchais à les suivre par la suite avec ma casquette de bénévole ou à demander des informations sur leurs comptes aux bénévoles qui les recevaient ou pendant la réunion collective. Cet exercice était un bon entraînement pour réfléchir à mes propres représentations. En outre, il m'a aidé à repérer comment les individus géraient leurs performance et mise en récit face aux bénévoles de l'accompagnement individuel censés les aider et comment - et si - leurs récits se modifiaient dans les coulisses de l'accueil (Goffman, 1973).

Je m'approche de Noah qui n'a visiblement pas envie de me parler. Il veut voir un expert, car « il ne va pas bien », me dit-il, «il a besoin de soins urgents. » Il clôt ainsi notre conversation. Quelques minutes plus tard, il revient me chercher : il ne peut pas trop attendre, il ne devrait pas partir trop tard. Ses mots et son regard mêlent urgence et fatigue. Johan s'est libéré, il peut le recevoir. Je profite et je m'ajoute au duo. Noah entre, fouille dans son sac pour en sortir des papiers abîmés - un ordre de quitter le territoire, une attestation de résidence datée, un certificat médical et un papier avec des adresses et numéros de téléphone probablement, des papiers journaux. Johan lui demande la raison qui l'amène. Noah dit qu'il a un enfant et veut le retrouver. Je le regarde, il ne m'a pas mentionné ce point auparavant. Des temporalités non chronologiques, des incohérences internes et des silences marquent la trame narrative de son discours biographique. Afin de se repérer dans son récit, Johan lui pose des questions précises : «vous êtes le père légitime de cet enfant ? Sa mère ? Est-elle française ? » Silence. Johan reprend : « vous voyez, nous avons besoin de comprendre quelle était la relation entre vous et à quand remonte ce que vous racontez, pour vous aider...» Noah nous fixe, puis il dit : « la maison est brûlée et moi je n'étais pas là, je suis arrivé trop tard, ils étaient déjà partis... » Johan a besoin de se repérer dans cette narration afin que son travail juridique soit efficace. Alors, il reprend ses questions et demande à Noah s'il a des documents d'identité. Il cherche plus explicitement de traces de sa présence préalable. Noah hausse la voie, comme pour nous signaler que nous ne prêtons pas assez d'attention à ce qu'il nous dit. À ce moment- 
là, il me regarde et, en m'indiquant, dit : « elle a tout pris, elle les avait avec elle à la maison... » Son interpellation inattendue provoque en moi une sensation de gêne, presque indescriptible, vis-à-vis de cet homme que je viens de rencontrer et à qui je n'ai pas pris de papiers. Je comprends que ma présence de femme blanche lui rappelle quelque chose, il évoque à travers moi des temporalités et personnes diverses. Johan fait des suppositions et lui demande si c'est une femme qui a ses documents d'identité quelque part ou s'ils étaient dans la maison qui a brûlé. Mais, Noah rapidement après dira : "quelqu'un m'a volé un autre sac, j'avais tout là-dedans. »

Ses réponses désarticulées ouvrent des espaces narratifs dont la solidité juridique est difficilement maîtrisable. Toutefois, bien que désorganisée de notre perspective, sa narration semble exploiter des connaissances vagues du droit migratoire, peut-être acquises par ses expériences et échanges préalables. En nous mentionnant ses attaches familiales par la paternité d'un enfant français, Noah signifie, en effet, qu'il pense satisfaire des conditions pour obtenir des papiers. Si auparavant en salle d'attente, il semblait appuyer son besoin d'aide par son corps malade et un besoin de soins ; après, dans l'interaction juridique, son corps était devenu celui du père d'un enfant français à sauver ou qu'il n'avait pas pu sauver. L'absence de documents pour soutenir ses propos et les contradictions dans son récit préoccupent Johan qui, au fur et à mesure, lui demande de plus en plus de détails factuels. Conformément à la loi, ce récit de présence doit se concrétiser par la production des preuves des liens familiaux. Noah sort des objets et d'autres papiers de son sac. Il en a effectivement beaucoup, mais, comme souvent les migrants en situation administrative irrégulière, il n'a pas « les "bons" documents qui [lui] sont réclamés et ceux qu'[il présente] ne font qu'accréditer la suspicion qui pèse sur la légitimité de son séjour» (Bernardot, 2012 : 98).

$\mathrm{Au}$ bout d'un moment, Johan se lève, son corps donne le signe que le temps de Noah est terminé. Il lui conseille de chercher les papiers avec les informations dont il nous parle et de revenir à nouveau. Je raccompagne Noah et, quand nous sommes seuls, il me dit : « si je le retrouve, j'irai mieux, mais je ne sais pas pour le sác, je ne sais pas. » Il se réfère à son fils ou peut être à son sac. Je me prends à penser : « qui pourra croire cet homme qui n'a pas de preuves et qui n'arrive pas à produire un récit logique ? »Comme il me dira après, Johan pense que Noah invente son récit et qu'il ést une de ces personnes « sans issue » dans le système actuel. Le travail d'encadrement de la psyché demandé désormais aussi aux intermédiaires du droit se heurte ici à une personne qui n'est pas docile et dont les troubles empêchent l'explication et la transmission de ce qu'il devrait faire pour devenir acceptable. En tant que chercheure, je ne suis pas à l'abri du procédé qui touche les bénévoles et qui leur enjoint le besoin de preuve et de véracité pour apporter de l'aide.

Dans le rituel de l'interaction d'aide, les corps, supports de la présentation de soi (Goffman, 1973), transmettent des informations précieuses, y compris concernant la psyché. Des tensions identitaires marquent le corps de Noah - qui ne veut pas partir sans obtenir quelque chose - et des discontinuités émotionnelles affectent sa psyché et fragmentent son récit. Comme Kobelinsky (2012 : 183-184) l'a observé pour les demandeurs d'asile, l'attente d'une réponse administrative, l'infantilisation dans le système d'accueil et l'inaction sont inscrites dans leurs corps et cristallisent la perte d'autonomie et l'incapacité décisionnelle de ces individus, ainsi que les dysfonctionnements de leurs liens sociaux et d'appartenance. Pour Noah, la situation de vide administratif qui perdure depuis longtemps, l'inactivité et d'autres événements, dont nous n'avons pas pu saisir la clé de lecture, affectent son corps - qui ressemble à celui d'une personne à la rue - et sa psyché - qui donne lieu à des troubles de conduite. Cependant, à travers nos échanges, son corps et sa psyché disparaissent en faveur de la recherche d'un récit et des documents. Noah adapte sa performance à cette dynamique et ne mentionne plus aucun malaise ni physique ni psychique. Seul Johan fait allusion à sa 
souffrance, lorsqu'avant de le quitter, l'invite délicatement à s'adresser à un service de santé mentale pour personnes étrangères.

La littérature a déjà montré qu'outre les corps utiles, car productifs, des travailleurs étrangers (Sayad, 1999), les corps des étrangers malades sont désormais devenus des corps exceptionnellement tolérés par les régimes migratoires (Musso, 2012 : 154 ; Fassin, 2001). Or, Noah ne pourra pas non plus s'appuyer sur une régularisation pour raisons de santé dont il ne pourrait pas prouver les fondements et la nécessité d'un traitement dans une structure française. Ainsi, son corps restera présent, bien qu'invisibilisé, sur le territoire français, et profondément marqué par les rapports utilitaristes et de domination définis par les politiques migratoires.

\section{CONCLUSION}

Au-delà de sa singularité (Passeron et Revel, 2005), le cas de Noah invite à penser la production des « récits de présence », entre droit et marginalité. Le rapport réflexif établi dans et sur le terrain m'a permis de repenser également ma posture, en tant que chercheure, et de cerner les potentialités et les limites de l'empathie pour collecter un récit cohérent et en comprendre les dynamiques sociales. Dans une permanence associative juridique, la coconstruction d'un tel récit, défendable, en vue de la régularisation administrative éclaire comment des personnes migrantes - oubliées dans les mailles des régimes migratoires deviennent le produit de ces mêmes régimes. D'une part, chez ces personnes, la précarisation administrative et ses temporalités créent des incohérences biographiques, des fragilisations intimes (Jamoulle, 2009) et l'impossibilité d'opérer une archéologie des traces de leur présence. La mémoire, fatiguée de et par l'expérience désenchantée de la migration, produit des dissonances identitaires et limite les opportunités de la mise en scène de soi demandée par les services de l'immigration et pré-demandée par les associations d'aide juridique. Ici, d'autre part, les critères imposés par l'État affectent les bénévoles qui agissent comme des intermédiaires du droit. Si pour favoriser la réussite de l'intermédiation, un partage narratif entre bénévoles et personnes migrantes en demande d'aide est souhaité, des représentations et des projections brident l'empathie pour laisser la place à des formes des suspicions similaires à celles collectives qui entourent la migration. Les échecs de la parole des personnes souffrantes et les réactions des intermédiaires participent d'une « société qui refuse à certains sujets une visibilité [sociale et administrative] qui, paradoxalement, implique en premier lieu d'être entendu » (Fœssel, 2009: 205). Or, les personnes migrantes dont traite cet article demandent une reconnaissance de leur présence, mais, également, un « droit à

l'incohérence ». Toutefois, leurs reconstructions narratives ne répondent pas aux normes des récits de présence définies par le dispositif d'immigration qui ne laisse aucun espace à l'incohérence biographique. C'est le corps que le dispositif d'immigration gouverne, et que le travail juridique et social encadre. Or, ici, les corps des étrangers ne sont ni utiles ni tolérables et leur psyché troublée est délaissée, car elle n'est pas rentable dans le mécanisme migratoire. L'adaptation du travail des intermédiaires à ces normes étatiques permet la poursuite d'un travail juridique dans le régime législatif en place, mais dépolitise ce travail et les récits des migrants en grande précarité, et désamorce leur potentiel de changement. La situation d'aide juridique montre, alors, les limites de la reconnaissance et dévoile les dynamiques ordinaires de la violence intersubjective qui, comme Butler (2007) le suggère, empêche l'individu de se revendiquer « soi-même ».

\footnotetext{
${ }^{1}$ Cette expression indique la succession des titres de séjour et des récépissés détenus par un étranger qui participent de sa stabilisation administrative et de son éventuelle obtention de nationalité.
} 


\footnotetext{
${ }^{2}$ Les extraits de notes de terrain et d'entretiens mobilisés dans l'article ne prétendent pas figer des situations, mais rendre compte des circonstances récurrentes bien exemplifiées par le cas choisi.
}

\section{Bibliographie}

Bernardot M. 2012, Captures, Marseille, Éditions du Croquant (coll. « Kritikos »).

Butler J. 2007, Le Récit de soi, Paris, PUF (coll. « Pratiques théoriques »).

Dal Lago A. 1999, Non-Persone: l'esclusione dei migranti in una società globale, Milan, Feltrinelli (coll. «Universale Economica - Saggi »).

Dauvergne C. 2008, Making People Illegal. What Globalization Means for Migration and Law (Law in Context), Cambridge, Cambridge University Press (coll. « Law in Context»).

Fassin D. 2001, «Quand le corps fait loi. La raison humanitaire dans les procédures de régularisation des étrangers » dans Sciences Sociales et Santé, n ${ }^{\circ}$, vol. 19 : 5- 34.

Fassin D., Bouagga Y., Coutant I., Eideliman J-S., Fernandez F., Fischer N., Kobelinsky C., Makaremi C., Mazouz S. et Roux S. 2013, Juger, réprimer, accompagner. Essai sur la morale de l'État, Paris, Le Seuil (coll. « Sciences humaines»).

Fassin D., Morice A. 2001, «Les Épreuves de l'irrégularité. Les sans-papiers, entre déni d'existence et reconquête d'un statut », dans Schnapper D. (éd.), Exclusions au cour de la cité, Paris, Anthropos-Economica, pp. 260-309.

Faavret-Saada J. 2009, Désorceler, Paris, Éditions de l'Olivier (coll. « Penser/rêver »).

Ferraris M. 2009, Documentalità. Perché è necessario lasciare tracce, Roma-Bari, Laterza (coll. « i Robinson / Letture »).

Fœssel M. 2009, « Le Mot pour se dire » dans Études, n9 9 , vol. 411 : 201-210.

Goffman E. 1973 [1 $1^{\mathrm{re}}$ éd. 1971], La Mise en Scène de la vie quotidienne/1. La Présentation de soi/2. Les Relations en public/3, Paris, Les Éditions de Minuit (coll. « Le sens commun »).

Jamoulle P. 2009, Fragments d'intime. Amours, corps et solitudes aux marges urbaines, Paris, La Découverte (coll. « Alternatives sociales »).

Kirmayer L. 2008, "Empathy and Alterity in Cultural Psychiatry" dans Ethos, n4, vol. 36: 457-474.

Kobelinsky C. 2012, « Des Corps en attente. Le quotidien des demandeurs d'asile» dans Corps, $\mathrm{n}^{\circ} 1$, vol. $10: 183-192$.

Hollan D. et Throop J. C. 2008, "Whatever Happened to Empathy?: Introduction" dans Ethos, $\mathrm{n}^{\circ}$ 4, vol. 36: 385-401.

Laacher S. 2009, Mythologie du Sans-papiers, Paris, Le Cavalier Bleu (coll. « MythO !»). 
Lhuilier D. 2005, « Le "Sale boulot” » dans Travailler, n¹4, vol. 2 : 73-98.

Martiniello M. et Rea A. 2011, « Des Flux migratoires aux carrières migratoires. Nouvelles perspectives empiriques et théoriques » dans SociologieS, en ligne :

http://sociologies.revues.org/index3694.html

Musso S. 2012, «Être régularisé au titre de la maladie en France » dans Corps, n 1 , vol. 10 : 153-161.

Passeron J-C. et Revel J. 2005, Penser par cas, Paris, Éditions de l'EHESS (coll. « Enquête »)

Pette M. 2014, «Associations : les nouveaux guichets de l'immigration ? Du travail militant en préfecture » dans Sociologie, ${ }^{\circ} 4$, vol. 5 : 405-421.

Sayad A. 1999, La Double absence. Des illusions de l'émigré aux souffrances de Vimmigré, Paris, Le Seuil (coll. « Sciences humaines, Liber»).

Spire A. 2005, Étrangers à la carte. L'administration de l'immigration en France, Paris, Grasset. 